\title{
Nachhaltigkeit als strategischer Imperativ für die Gesellschaft und Unternehmen
}

Heinz Simon Keil, Detlef Tietze

Was haben "Fridays for future" und Corona gemeinsam? Die Jugend fordert und die Pandemie erfordert einen nachhaltigen Umgang mit unseren Ressourcen, letztendlich zur Sicherung unseres Überlebens. Klimawandel, Verschmutzung, Reduzierung der Biodiversität, Ressourcenknappheit, Bevölkerungswachstum, Armut, Hunger, soziale Ungleichheiten, ungerechte Bildungschancen und Migration sind gewichtige Herausforderungen. Und es zeigt sich weltweit ein neues "globales" Bewusstsein für Nachhaltigkeit. Fridays for Future Protestbewegungen sind ein deutlich sichtbares Zeichen dafür. Und internationale Vereinbarung wie die UN Sustainable Development Goals (SDGs) oder das Pariser Klimaschutzabkommen sind ebenso ermutigende Zeichen für ein neues (kollektives) Bewusstsein.

Mit diesem kollektiven Bewusstsein geht verändern sich die Anforderungen an Unternehmen und deren Produckte und Services. Für Unternehmen wird Nachhaltigkeit zu einem strategischen Imperativ, der auf allen Ebenen des Geschäftsmodells verankert werden muss. Ein wichtiges Kennzeichen ist dabei die Zusammenarbeit der verschiedenen Stakeholder entlang der gesamten Wertschöpfungskette. Denn Nachhaltigkeit kann keine Gesellschaft und kein Unternehmen für sich allein lösen.

Keywords: Nachhaltigkeit; Kardashev; Kondratieff, Sustainability, Geschäftsmodell, Wertschöpfungskette, Multi-Stakeholder

\section{Nachhaltigkeit, Veränderungen, Voraussetzungen}

Die Corona-Krise ist die größte disruptive Veränderung, die wir in den letzten Jahrzehnten erleben müssen. Sie zeigt ungeschönt die Versäumnisse in den Gesellschaften und die Herausforderungen für die Menschheit auf. Hoffentlich erkennen alle, dass wir alle auf dem gleichen und einmaligen Planeten leben. Die Naturgesetze zeigen uns, dass auch wohl formulierte Grundgesetze außer Kraft treten. Der Geist steht nicht über der Natur.

Es macht Mut, dass die Gesellschaften angesichts akuter Bedrohungslagen durch Corona bereit sind, radikale Einschränkungen zu akzeptieren. Damit ist eine wesentliche Grundlage geschaffen, Lösungswege für die Herausforderungen anzugehen. 
Nachhaltigkeit ist ein Handlungsprinzip zur Ressourcen-Nutzung, bei dem eine dauerhafte Bedürfnisbefriedigung durch die Bewahrung der natürlichen Regenerationsfähigkeit der beteiligten Systeme (vor allem von Lebewesen und Ökosystemen) gewährleistet werden soll. Im entsprechenden englischen Wort "sustainable“ ist dieses Prinzip wörtlich erkennbar: to sustain im Sinne von aushalten bzw. ertragen. Mit anderen Worten: Die beteiligten Systeme können ein bestimmtes Maß an Ressourcennutzung dauerhaft aushalten, ohne Schaden zu nehmen. Wie kann ein gemeinsames, nachhaltiges Ziel formuliert werden. Können die Kardashev-Skala und der Entwicklungszyklus nach Kondratieff hier einen Rahmen geben?

\section{Energiewende als Basis für Nachhaltigkeit;}

Die Kardashev-Skala ist eine von dem russischen Astronomen Nikolai Kardashew 1964 vorgeschlagene Kategorisierung der Entwicklungsstufe extraterrestrischer Zivilisationen nach deren Energieproduktion und- verbrauch. Sie ist eine Methode zur Klassifizierung von Zivilisationen nach ihrem technologischen Niveau. Das Ziel war es, eine Skala für allen denkbaren Fortschritt zu entwerfen und unsere Zivilisation, wie auch jede andere vorstellbare Zivilisation, dort einordnen zu können. (1) Nach Kardashev verfügt eine Typ-0-Zivilisation über keine Energie, einer Typ-1-Zivilisation steht das gesamte Energiepotenzial ihres Planeten zur Verfügung.

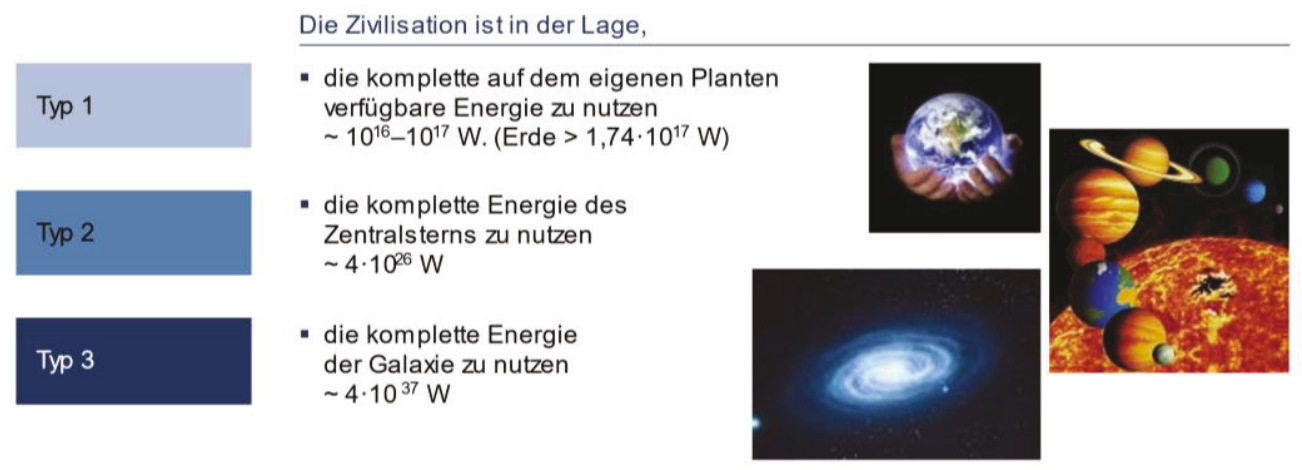

Abbildung 1: Der lange Weg zur Zivilisation 1.0

Der Energieverbrauch stieg von 24.500 TWh im Jahr 1950 auf rund 131.400 TWh im Jahr 2010; dabei verdoppelte sich der Pro-Kopf-Energieverbrauch. Bei gleicher Wachstumsrate des Pro-Kopf-Energieverbrauches und einem Anstieg der Weltbevölkerung auf über 9 Mrd. Menschen würde sich bis 2050 ein Energieverbrauch von über 350.400 
TWh ergeben. Um diesen Energiebedarf zu decken, wäre ein zusätzliches Leistungsäquivalent von etwa 48.000 fossilen Kraftwerken mit je 500 MW, 24.000 Kernkraftwerken mit je $1000 \mathrm{MW}$ oder 150.000 km² Photovoltaikanlagen notwendig.

Können wir so weitermachen?

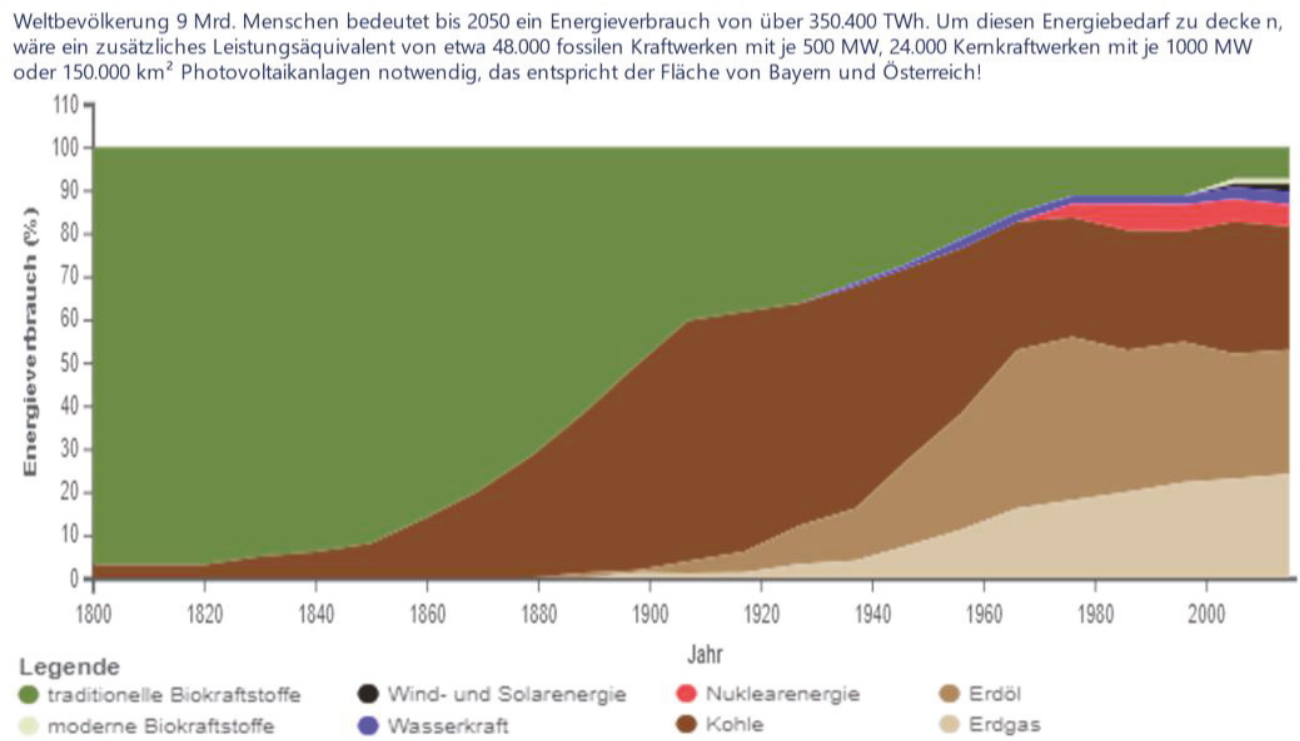

Abbildung 2: Energiesplit der Erde

Die Idee von Europa, in ihrem besten Sinne, ist ein Meilenstein auf dem Weg zu Typ 1, weil die europäische Idee ebenfalls auf gemeinsamen Interessen und Zielen beruht. Die Digitalisierung ist, ebenso wie die Energiewende, einer der treibenden Faktoren für die Möglichkeit, weiter im Rahmen dieser Skala voranzuschreiten. Eine Typ-1-Zivilisation muss sich auch bewusst für das Leben und die Zukunft entschieden haben und allzu oft scheinen wir uns aktuell rückwärtszubewegen, denn nationalstaatliche Interessen haben Auftrieb.

\section{Nachhaltige Struktur-/Konjunkturzyklen}

Bei der Suche nach einer Antwort auf die Frage zur Entstehung langfristiger Strukturzyklen gelangt man unweigerlich zum Ökonom Nikolai Kondratieff. Er beobachtete langfristige Wirtschaftsschwankungen mit Zyklen von 40 bis 60 Jahren und entwarf die sog. Kondratieff-Zyklen. Nach seiner Theorie stehen am Anfang eines jeden Zyklus 
neue technologische Errungenschaften, die zu Trägern eines langanhaltenden Konjunkturaufschwungs werden. Voraussetzung ist, dass diese Basisinnovationen nahezu alle Bereiche der Volkswirtschaft durchdringen und dadurch in der gesamten Wirtschaft neue Produktivitätsschübe auslösen. Seit der industriellen Revolution Ende des 18. Jahrhunderts bis heute unterscheidet er zwischen sechs Kondratieff-Zyklen.

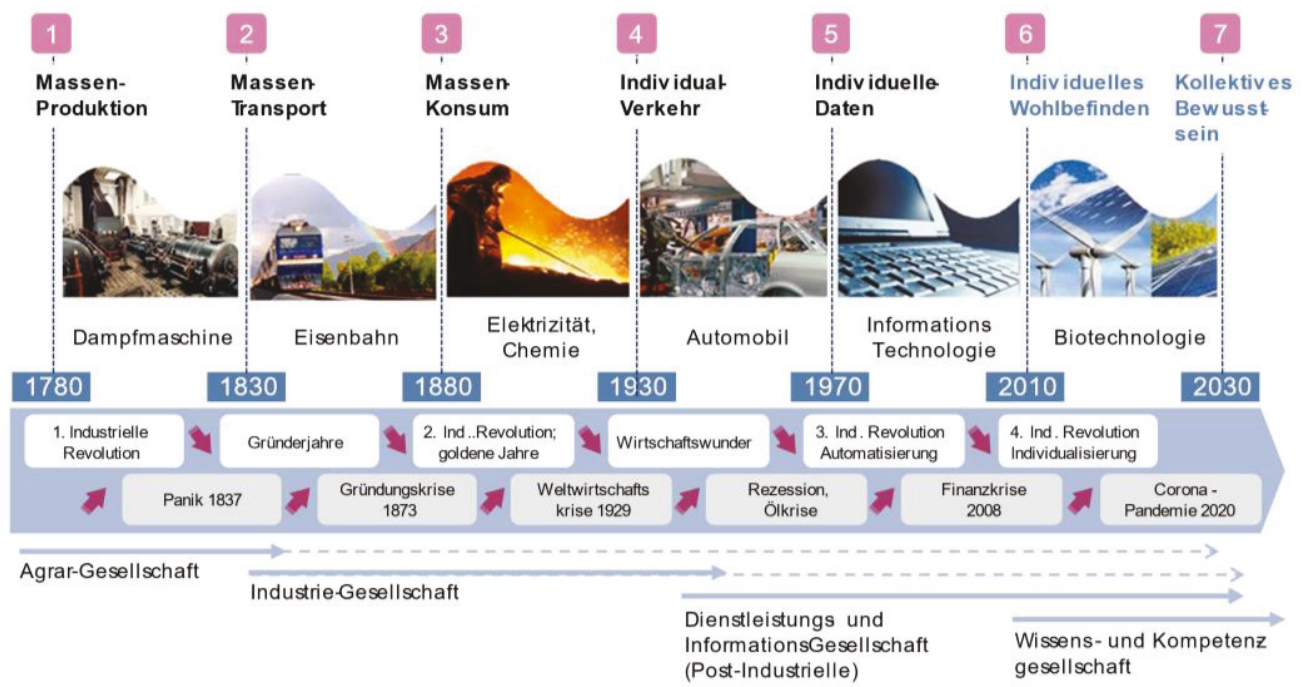

Abbildung 3: Kollektives Bewusstsein der nächste Kondratieff Zyklus?

Ein langer Strukturzyklus kommt zu seinem Ende - Mitauslöser ist wohl der CoronaVirus. Noch mehr Investitionen können die Produktivität nicht mehr steigern.

Nach Kondratieff führen nur Investition in den "Flaschenhals" aus der Krise. D.h. der knappste Produktionsfaktor muss produktiver gemacht werden. Dieser "Flaschenhals" sind qualifizierte, kompetente Wissensträger, die es ermöglichen die Digitalisierung anzuwenden und umzusetzen.

Eine neue auf digitale Prozesse aufbauende Bildungsinitiative von der Kita bis zum Rentner schafft die Basis den Flaschenhals zu eliminieren und den Weg in Richtung Zivilisation 1.0 und der Kompetenzgesellschaft anzugehen um damit für den Zyklus eines kollektiven, gesellschaftlichen Bewusstseins gewappnet zu sein.

Und - die Produktivität zu heben liegt ja tief in den "Genen" unseres Wirtschaftssystems leider nicht in unseren Verwaltungen und schon gar nicht in unsrer Bildung. Deshalb wird die Industrie mit seinen Ansätzen zu substanzieller, nachhaltiger Verbesserung als strategischen Imperativ voranschreiten müssen. 


\subsubsection{Agrar- und Industrie-Gesellschaft}

Der globale Wandel bringt für den Agrar- und Ernährungssektor insgesamt sowie speziell für die Agrarentwicklung und die Welternährung zahlreiche Herausforderungen mit sich. Hierzu gehören die gesellschaftlichen Anforderungen an eine multifunktionale Landwirtschaft, die zunehmende Verknappung der Produktionsfaktoren Boden und Wasser, sich verändernde Preisrelationen zwischen Biomasse und fossilen Energieträgern, sowie die erwarteten Auswirkungen des Klimawandels. Um diesen Herausforderungen zu begegnen, bedarf es privatwirtschaftlicher Initiative, leistungsfähiger öffentlicher Institutionen sowie eines hohen zivilgesellschaftlichen Engagements und eines Umbaus der Agrar- und Ernährungspolitik.
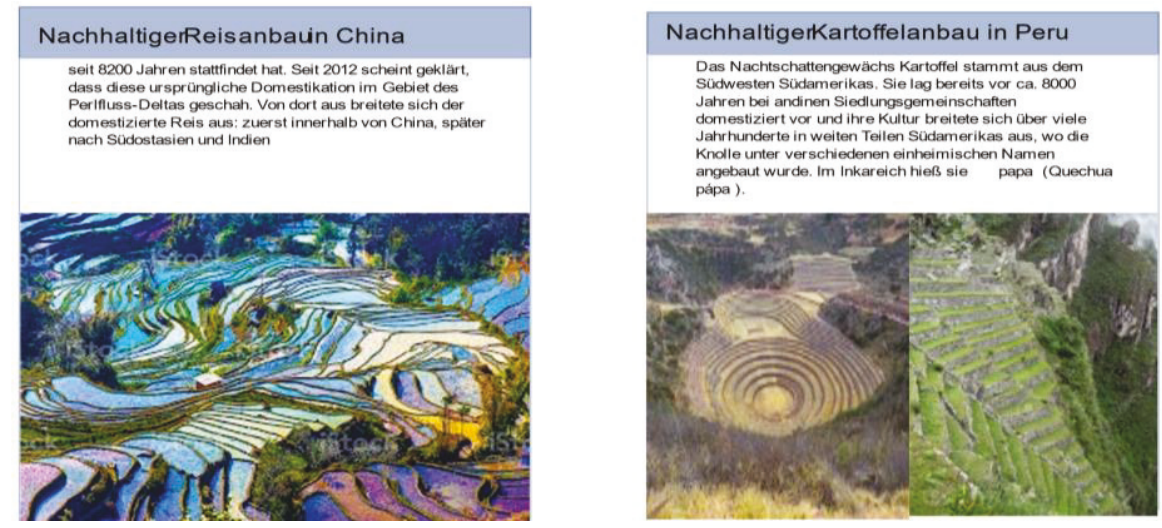

Abbildung 4: Nachhaltigkeit in der Agrarwelt

Die Entwicklung der Industriegesellschaft in den Schritten Mechanisierung (Industrie 1.0), Massenproduktion (Industrie 2.0), Automatisierung (Industrie 3.0) geht nun über das Internet der Dinge und Dienste in die vierte industrielle Revolution: Industrie 4.0. Die intelligente und dauerhafte Verknüpfung und Vernetzung von Maschinen, Menschen und maschinell betriebenen Abläufen in der Industrie, sie macht Produktion individueller und effizienter. Wichtigster Aspekt für die Zukunft ist dabei die Dezentralisierung der Entscheidungen, das heißt, sowohl Mitarbeiter als auch Maschinen und Produkte kommunizieren miteinander. 


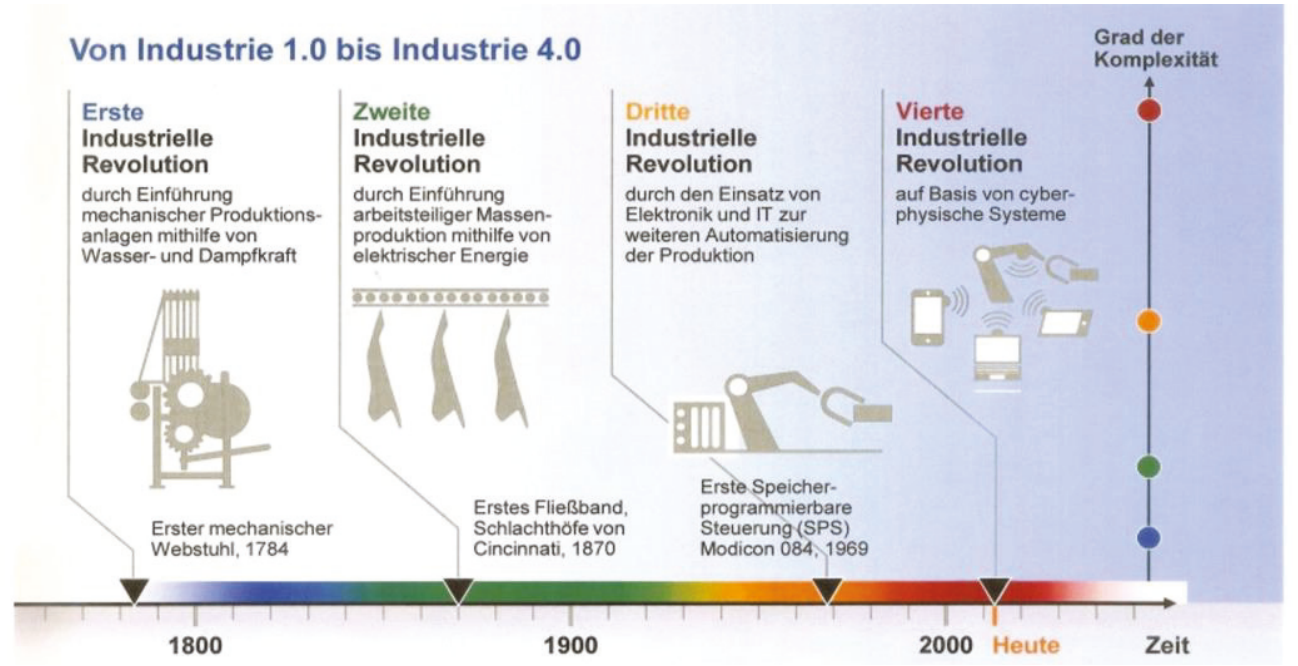

Abbildung 5: Die Revolutionen der Industrie-Gesellschaft

\subsubsection{Dienstleistungs- und Informations-Gesellschaft}

Die Informationsgesellschaft ist Ausdruck des Megatrends, dass weltweit, vor allem natürlich in den Industriestaaten, messbare Informationen, große Datenmengen, Big Data quantitativ und - nach entsprechender Auswertung - qualitativ immer wichtiger werden. Die Begriffe Informations-, Wissens- und Kompetenzgesellschaft werden oftmals synonym oder in ähnlicher Bedeutung benutzt.

\subsubsection{Wissens- und Kompetenz-Gesellschaft}

Der Begriff Wissensgesellschaft bezeichnet eine Gesellschaftsformation in hochentwickelten steht in engem Zusammenhang mit den Begriffen Informationsgesellschaft und Netzwerkgesellschaft sowie Ländern, in der individuelles und kollektives Wissen und seine Organisation vermehrt zur Grundlage des sozialen und ökonomischen sowie des medialen Zusammenlebens werden. Allerdings baut grundsätzlich jedes gesellschaftliche System auf Wissen auf, aber vor allem auf die Kompetenzen seiner Bürger.

Eine Wissensgesellschaft zeichnet sich auch dadurch aus, dass möglichst viele Bürger über Voraussetzungen verfügen, die es innen erlauben, das Angebot an Informationen kritisch und uneingeschränkt zu nutzen, um sich ein eigenes Urteil im Sinne eines vernünftigen Arguments bilden zu können. 


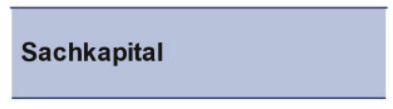

Hierarchie/Kontrolle

Vertikale Kommunikation

Sequentielle Prozesse

Produkte

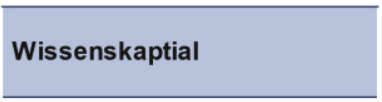

Vernetzung/Fokussierung

Horizontale Kommunikation

Simultane Prozesse

Problemlösungen

\section{Kollektivfähigkeiten}

Interaktion

Teamorientierte Kommunikatior

Algorithmen

Nachhaltiges Systemdenken

Abbildung 6: Von der Informationsgesellschaft zur Kompetenzgesellschaft

Wissensarbeit ist dadurch gekennzeichnet, dass das relevante Wissen

- kontinuierlich revidiert,

- permanent als verbesserungsfähig angesehen,

- prinzipiell nicht als Wahrheit, sondern als Ressource betrachtet wird und

- untrennbar mit Nichtwissen gekoppelt ist.

Wissensarbeit zielt:

- auf Wissensgenerierung, diese zielt

- auf Innovation, diese

- auf die Sicherung und Stärkung der Wettbewerbsfähigkeit von Unternehmen unter den verschärften Anforderungen globalisierter Märkte.

\subsubsection{Kompetenz-Gesellschaft (Kollektiv-Gesellschaft)}

Reines Wissen allein ist aber noch kein Garant für erfolgreiche Problembewältigung. Es geht also um die Befähigung, auf der Grundlage und mit Hilfe dieses Wissens selbstbestimmt, selbstorganisiert, kreativ und innovativ zu handeln?

Die Kreativität, also das, was wir aus den Tatsachen machen, ist nicht im Internet. Sie beruht auf individuellen und kollektiven Fähigkeiten. Dort wo wir Wissen verknüpfen, dort entstehen Assoziationen, Ideen. Die Vorstellung, man brauche nichts mehr im Kopf zu haben, weil man alles nachschlagen könne, ist falsch. Jedenfalls dann, wenn 
es einem nicht um die Reproduktion, sondern um die Produktion von Wissen geht." [4]

Zu Ende gedacht, bedeutet dies nicht mehr und nicht weniger als die Umwandlung der Wissensgesellschaft in eine Kompetenzgesellschaft - in eine Gesellschaft, in der möglichst viele befähigt werden, selbstorganisiert und kreativ zu handeln.

So kann man zusammenfassen, dass Kompetenzen gefragt sind,

- die sich schnell verändernden Rahmenbedingungen, wechselnden Anforderungsprofilen und Problemen anzupassen,

- die Fähigkeit ausmachen, das, was man in einem Sachbereich weiß und kann, auch auf andere, fremde Sachbereiche zu übertragen,

- die darüber hinaus in die lage versetzen, das gelernte auf Ungelerntes anzuwenden und

- die ganz allgemein bedeuten, gewohnten Umgang mit Problemen und ihrer Lösung zu haben.

Kompetenz besagt damit, dass Wissen und Können eine Einheit bilden. Auf diese Kompetenzen hin müssen Bildung und Ausbildung in Zukunft organisiert werden. Damit sind wir auf dem Weg in eine Kompetenzgesellschaft.

Daraus ergibt sich folgende verkürzte Zusammenfassung: „Information ist der Rohstoff, Wissen der Stoff, Kompetenz das Ziel für eine moderne Gesellschaft und dazu benötigen wir ein nachhaltiges, fähigkeitsförderndes Bildungssystem“. Von der Informationsgesellschaft zur Wissensgesellschaft, von der Wissensgesellschaft zur Kompetenzgesellschaft verläuft die gesellschaftliche Entwicklung, ohne andere Entwicklungsziele, andere Megatrends zu negieren.

Die beschrieben langfristigen technologischen Zyklen zeigen aber eine übergeordnete Tendenz; die ersten drei Zyklen halfen Massenbewegungen zu unterstützen, die Zyklen vier bis sechs unterstützen die Menschen in ihrer individuellen Ausprägung. Und was werden die nächsten Zyklen als gemeinsamen Nenner haben?

Hypothese: Es wird ein kollektivistisches, ganzheitliches Bewusstsein entstehen. Mit der Erkenntnis, dass wir alle gemeinsam nur einen Planeten haben. 


\section{Der Weg zur substanziellen Nachhaltigkeit als strategischer Imperativ}

\section{Steigender Druck zur Verankerung von Nachhaltigkeit in Unternehmen und Produkten}

Ein verändertes gesellschaftliches Bewusstsein für die aufgeführten Herausforderungen unserer Zeit führt dazu, dass sich die Rahmenbedingungen und Anforderungen an Unternehmen rapide verändern. Stakeholder haben erhöhte Anforderungen an Nachhaltigkeit von Produkten und Geschäftspraktiken der Unternehmen:

- Klimaschutzforderungen und die Einhaltung sozialer Standards spielen in gesellschaftlichen Diskussionen und gesellschaftlicher Aufmerksamkeit eine immer größere Rolle; nicht zuletzt lassen sich Missstände in digitalen und viralen Kampagnen schnell verbreiten und üben so Druck auf Unternehmen auf

- Kundenerwartungen verändern sich rapide, Kunden wollen verstärkt nachhaltige Produkte und Dienstleistungen konsumieren und ihren eigenen ökologischen Fußabdruck verbessern

- Investoren und Kapitalmärkte schwenken um, Umwelt- und Klimaauswirkungen werden als Geschäftsrisiken für Investments interpretiert; das hat Auswirkungen auf Kapitalflüsse und Finanzierungskonditionen für Unternehmen, es findet eine enorme Umlenkung von Kapital statt, das immer stärker durch die Berücksichtigung Nachhaltigkeitskriterien allokiert wird

- Eine intrinsisch motivierte Generation von Mitarbeitern kommt in die Unternehmen, die ihre Erwartungen an Nachhaltigkeit auf das Unternehmen übertragen; daher wird Nachhaltigkeit von Produkten und Geschäftspraktiken wichtig, um neue Mitarbeiter und Talente zu rekrutieren und zu halten

- Weltweit nimmt die Anzahl von Gesetzen und Richtlinien mit Bezug zur Nachhaltigkeit zu, der regulatorische Druck steigt; darauf müssen sich Unternehmen einstellen und die Einhaltung der geforderten Standards sicherstellen.

Unternehmen werden sich dem Druck der verschiedenen Stakeholder nicht mehr entziehen können und müssen unter dem kumulativen Begriff der substanziellen Nachhaltigkeit ihre Geschäftspraktiken und Geschäftsprozesse anpassen. Damit verändert sich die Rolle und Bedeutung von Nachhaltigkeit. In den 1990er und 2000er Jahren war es noch eher "Beiwerk". Durch den beschriebenen Handlungsdruck und die wachsende Aufmerksamkeit der Stakeholder entwickelt sich Nachhaltigkeit von einer reinen Compliance und Desktop-Übung hin zu einem Faktor für die Schaffung von echten 
Wettbewerbsvorteilen. Nachhaltigkeit wird so zum strategischen Imperativ in der Ausrichtung von Unternehmen, Produkten und Geschäftspraktiken. Und die Pandemie bremst Nachhaltigkeit nicht aus, sondern ist ein Beschleuniger.

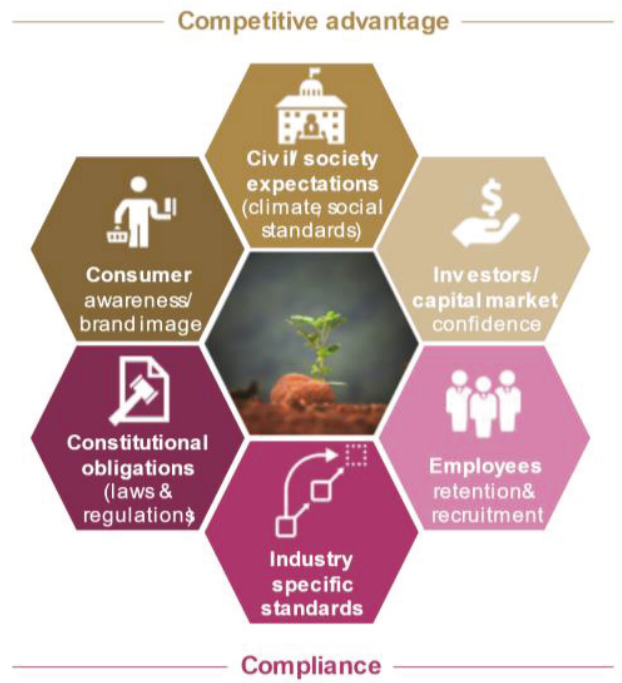

Abbildung 7: Framework zur Nachhaltigkeit

Die Definition von Nachhaltigkeit bleibt gleich...

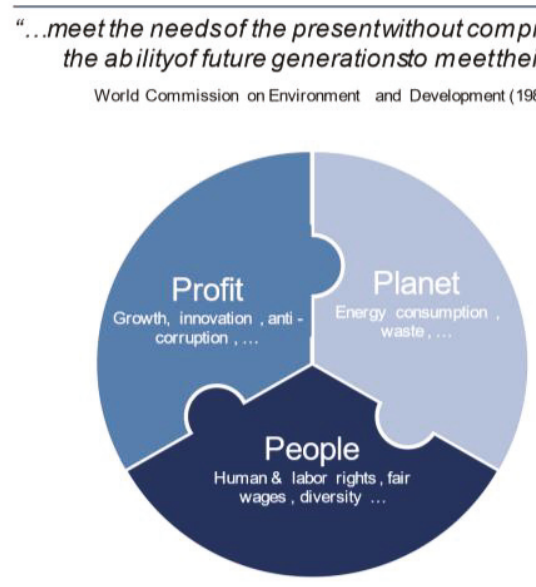

....aber die Herangehensweise hat sich seit den 90 er Jahren geändert

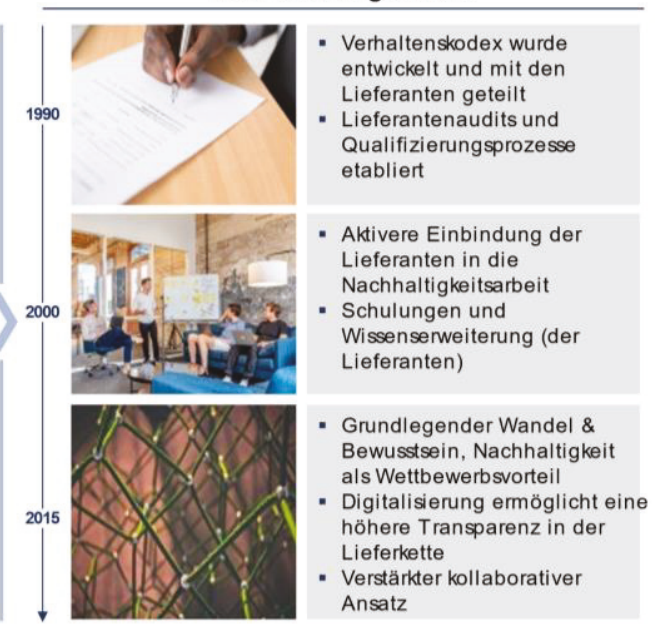

Abbildung 8: der Weg zum strategischen Wettbewerbsvorteil 


\section{Vorgehensmodell zur Verankerung von Nachhaltigkeit in Unternehmen und Produkten}

Die Verankerung einer substanziellen Nachhaltigkeit im Unternehmen erfordert die Integration verschiedener Nachhaltigkeitsaspekte in das gesamte Geschäftsmodell. Ein Vorgehensmodell zur Verankerung sollte die folgenden Aspekte berücksichtigen:

- Ausgangspunkt ist die die Abbildung von Nachhaltigkeit in der Unternehmensstrategie und den Zielsystemen; die Praxis zeigt, das substanzielle Nachhaltigkeit nur dann erfolgreich umsetzbar ist, wenn die Unternehmensführung voll dahintersteht und es durch Entscheidungen im täglichen Handeln das Kommittent erlebbar macht; Zur Strategie gehört auch eine eindeutige Begriffsdefinition von Nachhaltigkeit, welche Aspekte sollen unter dem Begriff subsummiert werden. Hier reicht die Bandbreite von Umweltgesichtspunkten über soziale Standards bis hin zu Fragen zum Umgang mit Korruption, Inklusion oder Diversity

- Nachhaltigkeit muss organisiert werden, d.h. es braucht eine Koordination und ein Governance-Mandat von Nachhaltigkeit zur übergreifenden Steuerung im Unternehmen; hier bieten sich dezidierte Stabsabteilungen an, aber vor allem geht es um einen engen Austausch zwischen allen Unternehmensfunktionen; zudem sollten entsprechende interne Regelwerke formuliert werden und mit Compliance-Überprüfungen die Einhaltung sichergestellt werden

- Bei der Verankerung von Nachhaltigkeitsaspekten in den Kernprozessen der Wertschöpfung geht es um die Berücksichtigung von Nachhaltigkeitsaspekten bei Produktentwicklung, Einkauf, Supply Chain, Vertrieb, Marketing und weiteren Funktionen bis hin zum Controlling. Die Nachhaltigkeitsziele der Unternehmensstrategie müssen auf die funktionalen Strategien der einzelnen Kernfunktionen heruntergebrochen und mit Zielen unterlegt werden; dann lassen sich nachhaltige Produkte und Wertschöpfungsketten realisieren.

- Identifizierung der Risiken: Voraussetzung für die Verbesserung der Nachhaltigkeits-Performance die Analyse der Risiken entlang der Kernprozesse und den Wertschöpfungsketten mit externen Lieferanten: In welchen Prozessschritten, Materialgruppen oder Lieferantenbeziehungen stecken die größten „Bedrohungen" und Chancen, die eigene Performance zu optimieren? Gleichzeitig hilft diese Analyse, die Aufgaben zu priorisieren und einen Startpunkt zu finden 
- Mit der Hilfe von digitalen Tools lässt sich Nachhaltigkeit in vielen Dimensionen unterstützen. Ob die Schaffung von Transparenz über Nachhaltigkeit in der Lieferkette, CO2-Kalkulationen oder Nachhaltigkeits-Reporting - mehr als 100 Lösungen sind am Markt inzwischen verfügbar

- Neben Digitalisierung ist Zusammenarbeit innerhalb des Unternehmens und Kollaboration über das eigene Unternehmen hinaus in Netzwerken und Partnerschaften ein wichtiger Faktor, um Nachhaltigkeit voranzubringen. Die Herausforderungen in Bezug auf Nachhaltigkeit kann kein Unternehmen für sich allein lösen. Es braucht die Integration der Partner entlang der Wertschöpfungskette und den Austausch mit anderen Unternehmen, um neue Impulse zu erhalten und die Geschwindigkeit in der Umsetzung von Nachhaltigkeit zu erhöhen; darüber hinaus lassen sich Skaleneffekte erzielen, wenn Unternehmen in Multi-Stakeholder-Initiativen Informationen und Wissen austauschen (z.B. Ergebnisse von Nachhaltigkeits-Audits bei Lieferanten)

- Als Fundament für substanzielle Nachhaltigkeit braucht es eine Transformation von Kultur und Mind-set in den Unternehmen. Die Sensibilisierung der Mitarbeiter, und die Befähigung durch Trainings und Ausbildung sind die Basis eine erfolgreiche Umsetzung von Nachhaltigkeit im Geschäftsmodell.
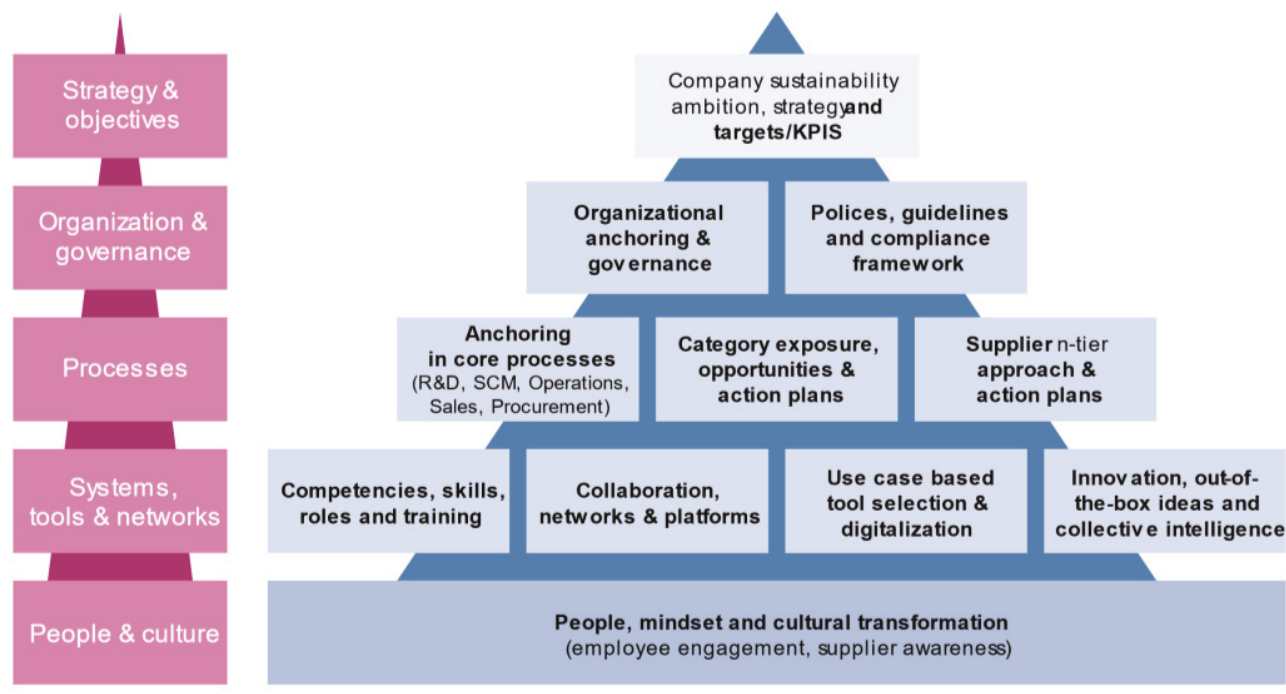

Abbildung 9: Bausteine des Framework 
Ein sichtbarer Ausdruck für ein kollektives Bewusstseins und enge Zusammenarbeit in der Praxis sind horizontale oder vertikale Multi-Stakeholder-Initiativen. Bei horizontalen Multi-Stakeholder-Initiativen vereinen sich Wettbewerber einer Branche, um gemeinsame Standards zu etablieren und bei der Einhaltung zusammenzuarbeiten. Bei vertikalen Initiativen schließen sich Unternehmen nachgelagerter Wertschöpfungsketten zusammen, also mit Einbindung der Lieferkette/Zulieferer.

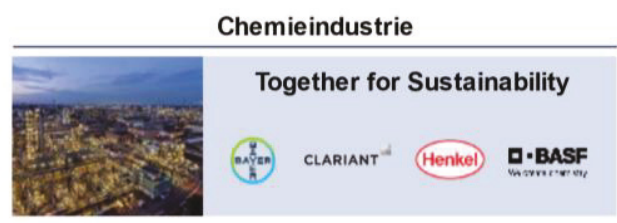

Bekleidungsindustrie

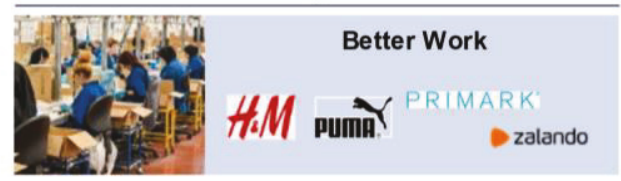

Industrieübergreifend

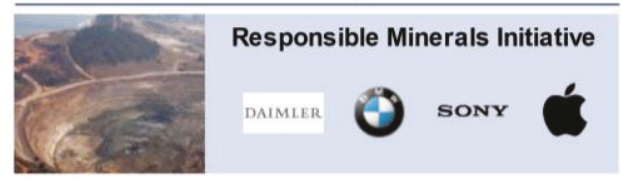

Süßwarenindustrie

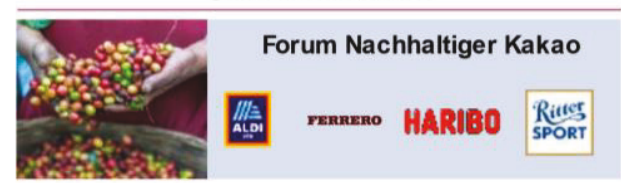

Lebensmittelindustrie

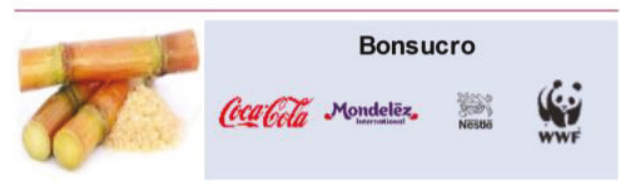

Lebensmittelindustrie/Drogerie

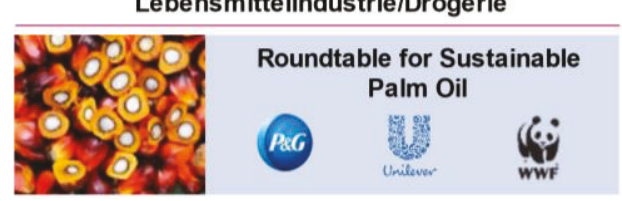

Abbildung 10: Stakeholder Initiativen

\section{Fazit: Nachhaltigkeit als strategischer Imperativ für Gesellschaft und Unternehmen - Konsequenzen für ein neues Werte-Lebenszyklus Management}

Die Geschwindigkeit von Klimawandel, Reduzierung der Biodiversität, Ressourcenknappheit, Bevölkerungswachstum sowie die mögliche Zunahme von sozialen Ungleichheiten, ungerecht verteilte Bildungschancen und Migration erfordern die Einleitung eines gesellschaftspolitischen, wirtschaftlichen, sozialpolitischen und technologischen Wandels, dessen Notwendigkeit durch die Corona-Pandemie zusätzlich unterstrichen wird.

Als Konsequenz braucht es ein neues Werte-Lebenszyklus Management, das Nachhaltigkeit als strategischen Imperativ für Gesellschaft und Unternehmen definiert. Die Voraussetzung für die Verankerung von Nachhaltigkeit in Gesellschaft und Unternehmen 
ist ein starkes kollektives Bewusstsein über die Herausforderungen und mögliche Lösungsansätze. Es braucht daher ein viel stärkeres ganzheitliches Systemdenken, das naturwissenschaftliche Erkenntnisse und technologogische Entwicklungen zusammenführt und in eine breitere gesellschaftliche und politische Verantwortung nimmt. Denn der rasante technologische und wissenschaftliche Fortschritt beschleunigt gesellschaftspolitische Zyklen und die Notwendigkeit gesellschaftlicher Anpassungen.

Die Auswirkungen auf sozialpolitische und gesellschaftliche Umfelder muss von Wissenschaft und Unternehmen den politischen Entscheidungsträgern nahegebracht werden und den Menschen bewusstgemacht werden. Denn bei der Corona-Krisen-Bewältigung sieht man ganz stark: Wer die Menschen aufklärt und bewusst einbindet, kann die Pandemie in Grenzen halten.

Die Geisteswissenschaften müssen intensiver in die von Algorithmen getriebene Transformation eingebunden werden, sonst verliert das breite Gemeinwesen den Anschluss an die Technologie! Was wir durch Corona vorgeführt bekommen, wie schwer sich Ämter und auch Schulen bei der Anwendung von notwendigen Technologien tun.

Die Umstellung auf ein naturnahes, nachhaltiges Engineering von Produkten, Systemen, Anlagen, Dienstleistungen und Geschäftspraktiken ist für Unternehmen und Gesellschaft eine disruptive Herausforderung. In ihrem Ausmaß, ihrer Reichweite und ihrer Komplexität wird es sich bei dieser Transformation um eine noch nie erlebte Erfahrung handeln. Was wir aber jetzt wissen, dass alle Teile des globalen Gemeinwesens, vom öffentlichen über den privaten Sektor bis hin zur akademischen Welt und der Zivilgesellschaft einbezogen sein müssen.

Die Ernährung der Welt, ganzheitliche Bildung, nachhaltiges Werte-Lebenszyklus Management und Kollektives Bewusstsein müssen die disruptiven Treiber der Zukunft sein, Voraussetzung ist ganzheitliches, nachhaltiges Systemdenken der Gesellschaft.

\section{Literaturverzeichnis}

(1) Nicola Armaroli, Vincenzo Balzani: Energy for a Sustainable World. From the Oil Age to a Sun-Powered Future. Weinheim 2011, S. $304 f$

(2) vonDaniel Domscheit-Berg ; Planetare Zivilisation - Frankfurter rundschau 29.03.2018

(3) Erik Händeler, auf XING, Beitrag zu Kondratieff-Zyklen

(4) Gerhard Willke: Globalisierung und Wissensgesellschaft, Auswirkungen auf Erwerbsarbeit und soziale Sicherung. In: Michael Bröning. Peter Oesterdieckhoff (Hrsg.): Deutschland in der globalen Wissensgesellschaft.Gutachten der FriedrichEbert Stiftung, 2004. 


\section{Kontakt}

Dipl.-Ing. Univ. Heinz Simon Keil

Design²Future

Fasanenstr. 5

93352 Rohr

Dr. Detlef Tietze

h \& z Unternehmensberatung AG

Max-Joseph-Straße 6

80333 München

detlef.tietze@huz.de 